\title{
Regorafenib treatment outcome for Taiwanese patients with metastatic gastrointestinal stromal tumors after failure of imatinib and sunitinib: A prospective, non-randomized, single-center study
}

\author{
CHIA-HSIANG HU ${ }^{1}$, CHUN-NAN YEH ${ }^{1}$, JEN-SHI CHEN ${ }^{2}$, CHUN-YI TSAI $^{1}$, \\ SHANG-YU WANG ${ }^{1}$, CHI-TUNG CHENG ${ }^{1}$ and TA-SEN YEH ${ }^{1}$ \\ Departments of ${ }^{1}$ Surgery and ${ }^{2}$ Medical Oncology, Chang Gung Memorial Hospital, \\ Chang Gung University, Kwei-Shan, Taoyuan 333, Taiwan
}

Received June 28, 2019; Accepted April 1, 2020

DOI: $10.3892 / \mathrm{ol} .2020 .11756$

\begin{abstract}
The present study aimed to conduct a prognosis analysis of Taiwanese patients with metastatic gastrointestinal stromal tumors (GISTs), who are resistant to or were unable to tolerate imatinib or sunitinib, and were subsequently treated with regorafenib. The study considered the survival, potential prognostic factors and safety of these Taiwanese patients. A total of 28 patients with pre-treated metastatic GIST, receiving regorafenib treatment, were analyzed between April 2014 and December 2017. Data were collected prospectively, and patients were followed up for a median of 14.8 months. It was reported that 50\% (10/20) of male patients and 50\% (4/8) of female patients demonstrated response and clinical benefit to regorafenib. The median progression-free survival (PFS) and overall survival (OS) time in all patients receiving regorafenib were 4.4 and 29.3 months, respectively. Good performance status and disease control mediated by regorafenib were independently associated with a more favorable PFS time. Good performance status, higher pre-treated albumin level, lower neutrophil:lymphocyte ratio (NLR) and lower platelet:lymphocyte ratio (PLR) were independent favorable predictors of OS time. Overall, poor performance status and
\end{abstract}

Correspondence to: Dr Chun-Nan Yeh, Department of Surgery, Chang Gung Memorial Hospital, Chang Gung University, 5 Fu-Hsing Street, Kwei-Shan, Taoyuan 333, Taiwan

E-mail: yehchunnan@gmail.com

Abbreviations: GIST, gastrointestinal stromal tumor; PFS, progression-free survival; OS, overall survival; NLR, neutrophil:lymphocyte ratio; PLR, platelet:lymphocyte ratio; PDGFR, platelet-derived growth factor receptor; IM, Imatinib mesylate; PR, partial response; SD, stable disease; TKI, tyrosine-kinase inhibitor; RECIST, Response Evaluation Criteria in Solid Tumors; ECOG, Eastern Cooperative Oncology Group; TTR, time to response; TTP, time to progression; HFSRs, hand-and-foot skin reactions

Key words: regorafenib, treatment outcome, GISTs poor disease control predicted a less favorable PFS time in Taiwanese patients with GISTs, who were pre-treated with regorafenib. Meanwhile poor performance status, high NLR, PLR and low albumin level predicted a less favorable OS time.

\section{Introduction}

Gastrointestinal stromal tumors (GISTs) are the leading mesenchymal neoplasms of the gastrointestinal system, with an annual incidence rate of 13.7 per million individuals in Taiwan (1). Effective systemic treatments for GISTs were not available globally until 2001 (2). However, identification of the involvement of constitutively active transmembrane receptor KIT and platelet-derived growth factor receptor A (PDGFRA) signaling in GIST oncogenesis justified the use of small-molecule tyrosine-kinase inhibitors for the treatment of GIST (3). Imatinib mesylate (IM) selectively inhibits several protein tyrosine kinases, such as the intracellular ABL kinase, the chimeric BCR-ABL fusion oncoprotein of chronic myeloid leukemia, KIT and PDGFRs (4-7). The expression of the cell-surface transmembrane receptor KIT, with tyrosine kinase activity, is a major diagnostic biomarker of GIST. The current understanding is that frequent gain-of-function mutations of KIT occur in GISTs (3), causing constitutive activation of KIT signaling and resulting in uncontrolled cell proliferation and resistance to apoptosis (3). For advanced GIST, IM treatment also exhibited favorable results in terms of progression-free survival (PFS) and overall survival (OS) time (8), and several clinical trials have also reported promising effects of this targeted therapy in increasing PFS and OS time (6,9-11).

Although IM has been known to result in notable improvements in the PFS and OS time of patients with GIST, partial response (PR) and stable disease (SD) was documented in 54\% of cases; however, $\sim 28 \%$ of patients will develop advanced or metastatic GIST $(5,6)$. The majority of patients with GIST will display drug resistance to imatinib and disease deterioration (12). A multi-target tyrosine-kinase inhibitor (TKI) that provides prolonged PFS time (27 weeks), compared with the placebo in a randomized phase III trial (13), was approved as the second-line targeted therapy for GIST after imatinib and 
sunitinib; however, resistance to sunitinib also developed (14). Thus, novel TKIs are needed as an alternative option for patients with GIST, in the event that resistance to sunitinib resistance develops.

Regorafenib is another multi-kinase inhibitor that antagonizes various targets, including KIT, PDGFRA, vascular endothelial growth factor receptor, RAF1, BRAF, RET and fibroblast growth factor receptor, in in vitro analyses (15). An international, multicenter, randomized, placebo-controlled, phase III trial (GRID) (16) reported that the median PFS time was 4.8 months for the regorafenib-treated group and this was longer compared with placebo group by 0.9 months. Based on the GRID study, regorafenib was then approved by the Food and Drug Administration in February 2013 for metastatic or unresectable GIST after resistance to imatinib and sunitinib had developed. Asian patients enrolled in the phase III GRID trial were from Japan, Korea, China and Singapore, therefore the present study investigated the efficacy of regorafenib in a Taiwanese population.

The present prospective, non-randomized, single-center study aimed to assess the efficacy, prognosis and safety of regorafenib in inducing an objective response or SD in population of individuals with advanced inoperable/metastatic GIST, who either developed resistance to or could not tolerate the toxicity associated with imatinib or sunitinib. In addition, a literature review was conducted to elucidate the effect of regorafenib on GIST globally.

\section{Materials and methods}

Patients, study design and efficacy evaluation. Between April 2014 and December 2017, 40 patients were diagnosed with advanced inoperable/metastatic GIST histologically (17) and received regorafenib therapy. The clinical data was collected prospectively and reviewed retrospectively. Of note, regorafenib treatment has been reimbursed by National Health Insurance in Taiwan since August 2016 (16). While 18 patients were enrolled from the previous trial (18), 22 were enrolled from the health reimbursement program. In the present study, however, only 28 patients who were refractory or intolerant to imatinib and sunitinib and with measurable disease based on the Response Evaluation Criteria in Solid Tumors (RECIST) version 1.1 (19) were eligible for further analysis. There were 20 males and 8 females with a median age of 61 years (range, 36-71 years). In addition, these 28 patients had an Eastern Cooperative Oncology Group (ECOG) performance score of $\leq 3$ and presented with adequate hepatic, renal and hematological functions. The dose of regorafenib was $160 \mathrm{mg}$ daily in a 3-week on/1-week off schedule, every 4 weeks, orally. Regorafenib was not stopped, unless unmanageable toxicity or disease progression occurred or consent was withdrawn. Of note, regorafenib could be continued in spite of documented disease progression if a clinical benefit was evident to the treating physician. In contrast, postponement of treatment or lowering of the dose was considered in the event of adverse drug-associated side effect and dose re-escalation was allowed after these side effects were resolved. Regular monthly check-ups of participants included routine physical examinations and evaluations of their performance status, weight, complete blood count and serum chemistry, including
Table I. Clinicopathological characteristics of patients with advanced GIST treated with regorafenib $(n=28)$.

\begin{tabular}{|c|c|c|}
\hline Characteristics & $\mathrm{n}$ & Range \\
\hline \multicolumn{3}{|l|}{ Median age at time of, years } \\
\hline Diagnosis of GIST & 52 & $28-66$ \\
\hline Diagnosis of metastasis & 52 & $30-67$ \\
\hline Start of imatinib & 52 & $30-68$ \\
\hline Start of sunitinib & 58 & $35-68$ \\
\hline Start of regorafenib & 61 & $36-71$ \\
\hline \multicolumn{3}{|l|}{ Sex, $\%$} \\
\hline Male/female & $20 / 8$ & $71.4 / 28.6$ \\
\hline \multicolumn{3}{|l|}{ ECOG, $\%$} \\
\hline $0-1 / 2-3$ & $22 / 6$ & $78.6 / 21.4$ \\
\hline \multicolumn{3}{|l|}{ Genetic mutation, \% } \\
\hline Exon 9 & 5 & 17.86 \\
\hline Exon 11 & 6 & 21.43 \\
\hline Exons 11 and 17 & 10 & 35.71 \\
\hline Exons 11, 13 and 17 & 5 & 17.86 \\
\hline Wild-type & 2 & 7.14 \\
\hline $\begin{array}{l}\text { Median length of imatinib } \\
\text { treatment, months }\end{array}$ & 63.23 & $9.4-155.54$ \\
\hline $\begin{array}{l}\text { Median length of sunitinib } \\
\text { treatment, months }\end{array}$ & 21.91 & $2.69-67.91$ \\
\hline \multicolumn{3}{|l|}{ Primary site, \% } \\
\hline Stomach & 4 & 14.26 \\
\hline Small bowel & 24 & 85.74 \\
\hline \multicolumn{3}{|l|}{ Metastatic site, $\%$} \\
\hline Liver & 20 & 71.43 \\
\hline Peritoneum & 18 & 64.29 \\
\hline Lung & 3 & 10.71 \\
\hline Others & 5 & 17.86 \\
\hline \multicolumn{3}{|l|}{ Prior failed TKI, \% } \\
\hline Imatinib & 28 & 100.00 \\
\hline Sunitinib & 28 & 100.00 \\
\hline Nilotinib & 2 & 7.14 \\
\hline
\end{tabular}

ECOG, Eastern Cooperative Oncology Group; TKI, tyrosine kinase inhibitor; GIST, gastrointestinal stromal tumor.

aspartate aminotransferase, alanine aminotransferase and total bilirubin to measure hepatic function, creatinine for renal function, and T3, T4 and thyroid stimulating hormone for thyroid function. Standard computed tomography scans for each patient were performed every 3 months. Tumor size was determined by measuring the diameter of $\geq 5$ target lesions and the largest dimension was used as a response evaluation indicator, according to the RECIST 1.1 criteria (19). Time to response (TTR=time point of the best response-time point of regorafenib administration) was defined as the interval for the best drug response during the treatment course. The time to progression (TTP=time point of disease progression-time point of regorafenib administration) was defined as the interval for the worse drug response with disease progression 
Table II. Antitumor response of advanced gastrointestinal stromal tumor treated with regorafenib $(n=24)$.

\begin{tabular}{|c|c|c|c|c|c|}
\hline Response & $\mathrm{n}(\%)$ & $\begin{array}{c}\text { Sex, } \\
\text { male/female, } \mathrm{n}\end{array}$ & $\begin{array}{l}\text { Median regorafenib } \\
\text { duration, months }\end{array}$ & $\begin{array}{l}\text { Median TTR/ } \\
\text { TTP, months }\end{array}$ & $\begin{array}{c}\text { Median OS, } \\
\text { months }\end{array}$ \\
\hline PR & 4 (14.29) & $3 / 1$ & 15.21 & 6.16 & 21.05 \\
\hline SD & $10(35.71)$ & $7 / 3$ & 6.08 & 2.11 & 9.54 \\
\hline PD & $10(35.71)$ & $8 / 2$ & 3.09 & 2.46 & 11.69 \\
\hline N/A & 4 (14.29) & $2 / 2$ & 0.46 & N/A & N/A \\
\hline
\end{tabular}

$\mathrm{PR}$, partial response; $\mathrm{SD}$, stable disease; $\mathrm{PD}$, progressive disease; N/A, not available; TTR, time to response; TTP, time to progression; OS, overall survival.

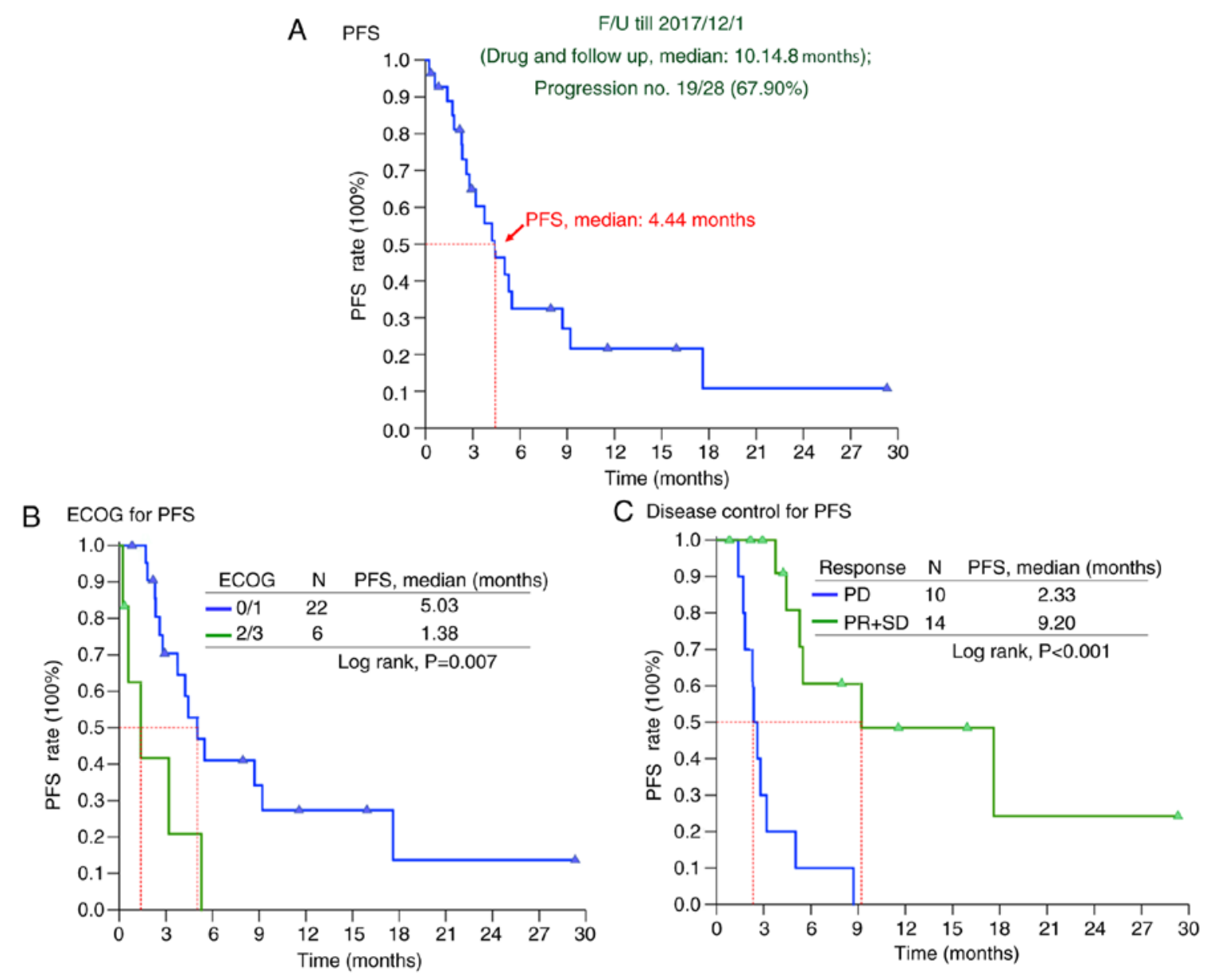

Figure 1. Survival analysis of PFS time. (A) Kaplan-Meier plot of the PFS time of 28 Taiwanese patients with advanced gastrointestinal stromal tumor treated with regorafenib, and in terms of (B) ECOG performance status and (C) response. PFS, progression-free survival; ECOG, Eastern Cooperative Oncology Group; F/U, follow-up; PD, progressive disease; PR, partial response; SD, stable disease.

during the clinical course. PFS was defined as no disease progression after start of regorafenib treatment. OS was defined as the survival after regorafenib administration, and the endpoint of the present study was either GIST-associated death or December 2017. A total of 4 patients were excluded from the survival analysis due to 2 of them having received regorafenib $<1$ month prior to enrolment in the present study, 1 withdrew due to severe and intolerable adverse events, and 1 was lost to follow-up. The adverse events of regorafenib were evaluated according to the National Cancer Institute Common
Terminology Criteria for Adverse Events version 4.0 (20). The protocol of the present study was approved by The Institutional Review Board of the Chang Gung Memorial Hospital (approval no. 103-6044A3; Taoyuan, Taiwan), and written informed consent was provided by all patients for drug administration and analysis of tumor-associated genetic alteration.

Analysis of KIT and PDGFRA mutations. Sections $(10-\mu \mathrm{m}$-thick) were prepared from formalin-fixed, 
F/U till 2017/12/1 (median: 10 months); Death no. 10/28 (35.70\%)

A os

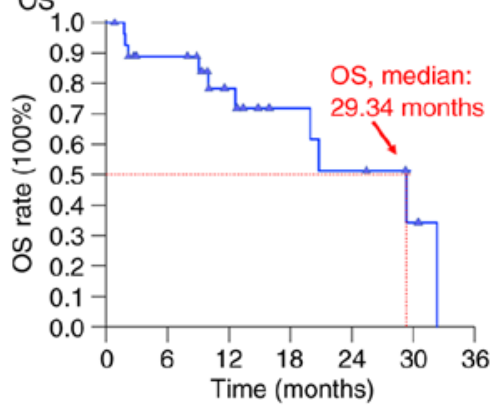

B

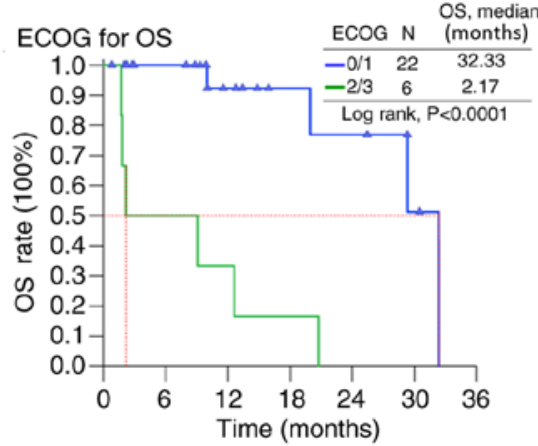

C Albumin for OS

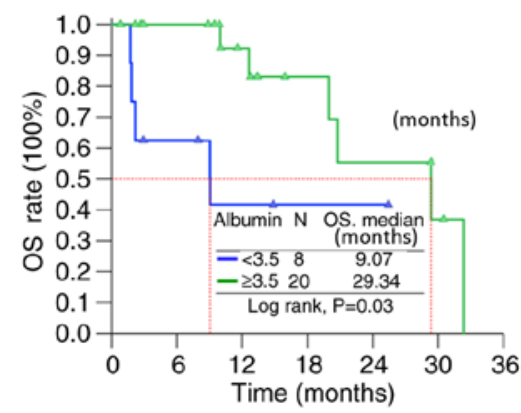

D NLR for OS

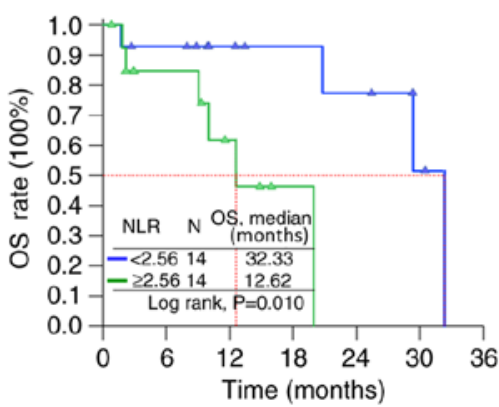

$E$

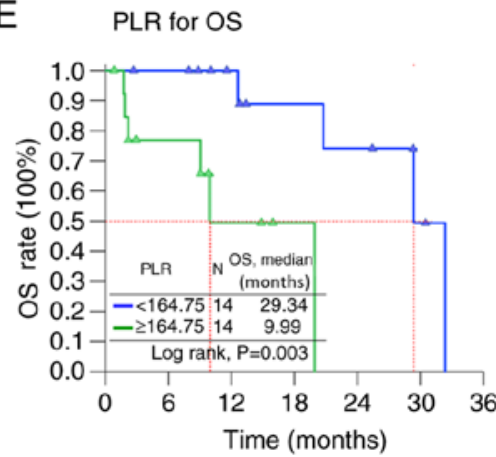

Figure 2. Survival analysis of OS time. (A) Kaplan-Meier plot of the OS time of 28 Taiwanese patients with advanced gastrointestinal stromal tumor treated with regorafenib, and in terms of (B) ECOG score, (C) albumin, (D) NLR and (E) PLR. ECOG, Eastern Cooperative Oncology Group; F/U, follow-up; NLR, neutrophil:lymphocyte ratio; PLR, platelet:lymphocyte ratio; OS, overall survival.

paraffin-embedded pretreatment specimens trimmed to enrich for tumor cells. Tissues were fixed with $10 \%$ formalin at room temperature for $\geq 24 \mathrm{~h}$. Subsequently, PCR was performed as previously described (21) on the DNA isolated from these sections to amplify the genomic DNA sequences of KIT and PDGFRA by Professor CY Tzen at Cathay Memorial Hospital (Taipei, Taiwan). Sequences for mutations of KIT and PDGFRA were analyzed as described previously (21).

Statistical analysis. For descriptive statistics, all the data are presented as percentage of patients or mean. Kaplan-Meier and log-rank tests were performed for time-to-event analysis. Several potential variables impacting long-term outcomes, including PFS and OS time, were analyzed for significance, including age ( $<61$ vs. $\geq 61$ years), sex, ECOG performance status (score 0-1 vs. 2-3), mutational status (presence vs. absence exon 17 mutation), response [complete response + PR vs. SD vs. progressive disease (PD)], primary site and metastatic site of GIST, and parameters of the following: White blood cells with differential counts [neutrophil:lymphocyte ratio (NLR)], platelet counts, platelet:lymphocyte ratio (PLR), and hemoglobin and albumin levels. All aforementioned factors were analyzed using a Cox multivariate proportional hazard model if statistical significance was identified using univariate analysis. An 'enter-selection' procedure was used to select the most relevant prognostic factors and only factors that remained significant were included in the final model. All statistical analyses were performed using SPSS version 20.0 (IBM Corp). $\mathrm{P}<0.05$ was considered to indicate a statistically significant difference.

\section{Results}

Clinical features. Table I summarizes the demographic characteristics of 28 patients (20 males and 8 females; median age, 61 years; range, 36-71 years) with advanced inoperable/metastatic GIST treated with regorafenib. All patients had received imatinib and sunitinib treatment prior to regorafenib, and $2 / 28(7.14 \%)$ had also received nilotinib. The median length of imatinib treatment was 63.23 months (range, 9.4-155.54 months) and that of sunitinib treatment was 21.91 months (range, 2.69-67.91 months). Most of the patients had a favorable ECOG score $(22 / 28 ; 78.6 \%)$. While the small bowel was the leading primary site for GISTs treated with regorafenib $(24 / 28 ; 85.74 \%)$, the liver was the leading metastatic site $(20 / 28 ; 71.43 \%)$, followed by the peritoneum $(18 / 28$; $64.29 \%)$ and lungs $(3 / 28 ; 10.71 \%)$. Out of the 28 patients with GIST with mutation data, exons 11 and 17 were the most common $(n=10)$, followed by exons 11,13 and $17(n=5)$, exon 9 $(n=5)$, exon $11(n=6)$ and wild-type $(n=2)$.

Treatment and outcomes. Regorafenib was administered to patients with pretreated metastatic GISTs, a starting dose of $160 \mathrm{mg}$ /day was administered to all 28 patients. All patients were followed up after regorafenib administration at regular intervals until death or until December 2017. Table II summarizes the best antitumor response of regorafenib of all patients with pretreated metastatic GIST. Overall, 24/28 patients were available for the efficacy evaluation, four (14.29\%) demonstrated a PR, 10 (35.71\%) SD and $10(35.71 \%)$ PD. In addition, $50.00 \%$ of patients with GIST exhibited a clinical benefit. Of 24 patients, the median TTR for four patients who presented PR and 10 SD were 6.2 and 
Table III. Prognostic analysis for the PFS time for patients with gastrointestinal stromal tumor using the univariate and multivariate model.

\section{Univariate}

Multivariate

Variable

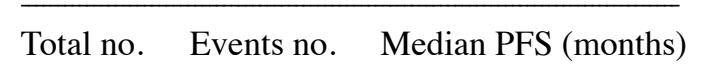

$\begin{array}{cc}\text { Hazard } \\ \text { Log-rank P-value } & \text { P-value } \\ \text { ratio }(95 \% \mathrm{CI})\end{array}$

Age, years

$\leq 61$
$>61$

Sex

Male

Female

ECOG

$0-1$

2-3

Genetic status

Non-exon 17

Exon 17

Metastatic site

Non-liver

Liver

Total lymphocyte count

$<1,550$
$\geq 1,550$
NLR

$<2.56$

$\geq 2.56$

PLR

$<164.75$

$\geq 164.75$

Albumin

\section{$<3.5$}

$\geq 3.5$

Response

$$
\text { PR + SD }
$$

PD

\section{5}

13

20

8

22

6

13

15

\section{8}

20

14

14

14

14

14

14

8

20

.

14

10
12

7

13

6

14

5

\section{4}

15

CI, confidence interval; ECOG, Eastern Cooperative Oncology Group; PLR, platelet:lymphocyte ratio; NLR, neutrophil:lymphocyte ratio; $\mathrm{PR}$, partial response; $\mathrm{SD}$, stable disease; $\mathrm{PD}$, progressive disease.
0.378

0.125

5.29

2.33

$\begin{array}{lll}0.007 & 0.009 & 4.330\end{array}$

(1.434-13.069)

5.03

24

(n)

1.38

$<0.0001$

0.001

8.326

(2.513-27.588)

\section{9}

10

\section{8}

11

12

7

11

8

5

14

3.19

5.30

0.136

9.20

3.75

0.187

8.70

3.19

0.655

3.75

5.29

0.993

3.75

5.29

0.403

4.24

4.44

9.20

2.33

0.422

6

10 
Table IV. Prognostic analysis for the OS time of patients with gastrointestinal stromal tumor using the univariate and multivariate model.

\begin{tabular}{|c|c|c|c|c|c|c|}
\hline \multirow[b]{2}{*}{ Factors } & \multicolumn{3}{|c|}{ Univariate } & \multicolumn{3}{|c|}{ Multivariate } \\
\hline & Total, $\mathrm{n}$ & Events,n & Median OS, months & Log-rank P-value & P-value & Hazard ratio $(95 \% \mathrm{CI})$ \\
\hline Age, years & & & & 0.321 & & \\
\hline$\leq 61$ & 15 & 6 & 32.33 & & & \\
\hline$>61$ & 13 & 4 & 29.34 & & & \\
\hline Sex & & & & 0.573 & & \\
\hline Male & 20 & 8 & 20.76 & & & \\
\hline Female & 8 & 2 & N/A & & & \\
\hline ECOG & & & & $<0.0001$ & 0.001 & $\begin{array}{c}15.053 \\
(3.024-74.929)\end{array}$ \\
\hline $0-1$ & 22 & 14 & 32.33 & & & \\
\hline $2-3$ & 6 & 6 & 2.17 & & & \\
\hline Genetic status & & & & 0.049 & 0.065 & $\begin{array}{c}3.723 \\
(0.92-15.056)\end{array}$ \\
\hline Non-exon 17 & 13 & 6 & 20.76 & & & \\
\hline Exon 17 & 15 & 4 & 32.33 & & & \\
\hline Metastatic site & & & & 0.117 & & \\
\hline Non-liver & 8 & 1 & N/A & & & \\
\hline Liver & 20 & 9 & 19.94 & & & \\
\hline Total lymphocyte count & & & & & 0.382 & \\
\hline$<1,550$ & 14 & 5 & 19.94 & & & \\
\hline$\geq 1,550$ & 14 & 5 & 32.33 & & & \\
\hline NLR & & & & 0.010 & 0.033 & $\begin{array}{c}10.876 \\
(1.217-97.211)\end{array}$ \\
\hline$<2.56$ & 14 & 4 & 32.33 & & & \\
\hline$\geq 2.56$ & 14 & 6 & 12.62 & & & \\
\hline PLR & & & & 0.003 & 0.019 & $\begin{array}{c}13.543 \\
(1.544-118.822)\end{array}$ \\
\hline$<164.75$ & 14 & 4 & 29.34 & & & \\
\hline$\geq 164.75$ & 14 & 6 & 9.99 & & & \\
\hline Albumin & & & & 0.03 & 0.045 & $\begin{array}{c}4.221 \\
(1.033-17.246)\end{array}$ \\
\hline$<3.5$ & 8 & 4 & 9.07 & & & \\
\hline$\geq 3.5$ & 20 & 6 & 29.34 & & & \\
\hline Response & & & & 0.172 & & \\
\hline $\mathrm{PR}+\mathrm{SD}$ & 14 & 3 & 29.34 & & & \\
\hline PD & 10 & 4 & 19.94 & & & \\
\hline
\end{tabular}

CI, confidence interval; ECOG, Eastern Cooperative Oncology Group; PLR, platelet:lymphocyte ratio; NLR, neutrophil:lymphocyte ratio; $\mathrm{PR}$, partial response; $\mathrm{SD}$, stable disease; $\mathrm{PD}$, progressive disease; N/A, not available.

NLR and PLR (compared with high NLR and PLR) and good nutritional status with albumin $\geq 3.5 \mathrm{gm} / \mathrm{dl}$ were independent prognostic factors positively associated the OS time of patients with advanced inoperable/metastatic GIST after regorafenib treatment (Table IV and Fig. 2).

Literature review to compare the effect of regorafenib on GIST globally. For comparison with the present study cohort, a global literature review of patients with GIST who received regorafenib treatment was conducted $(16,22-25)$. The literature review (Table V) revealed that regorafenib exhibited similar clinical efficacy compared with the GRID trial (16) comprising of Asian patients, including Korean and Japanese, with advanced GIST who experienced treatment failure with imatinib or sunitinib. While the PFS time ranged between 4.4 and 13.2 months, the OS time ranged between 12.2 and 29.3 months. 


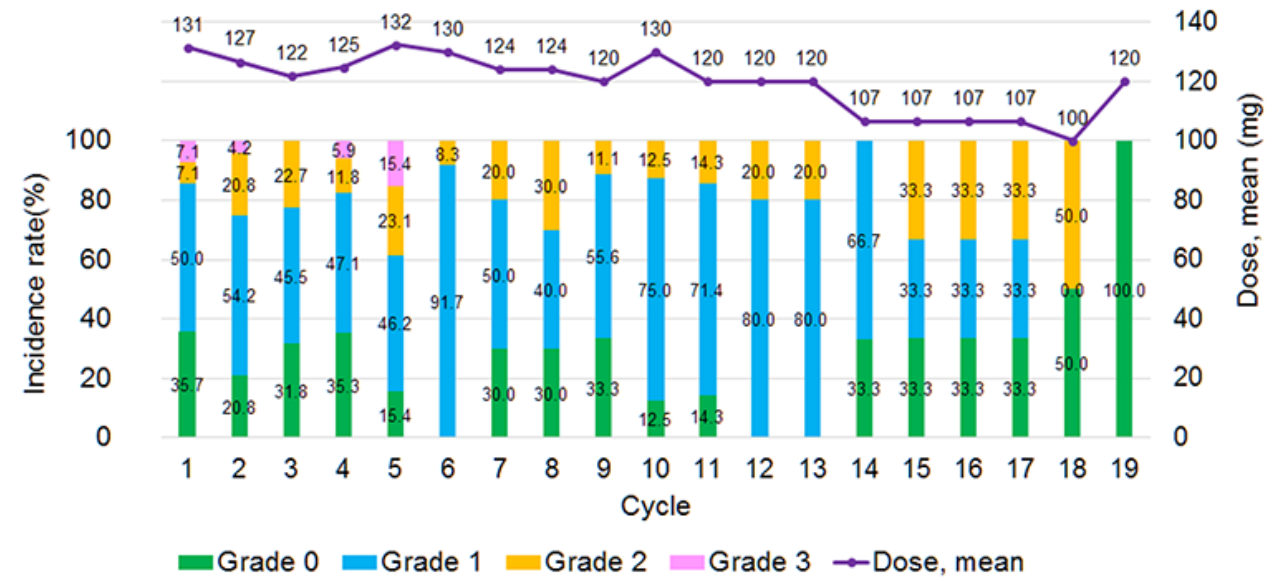

B

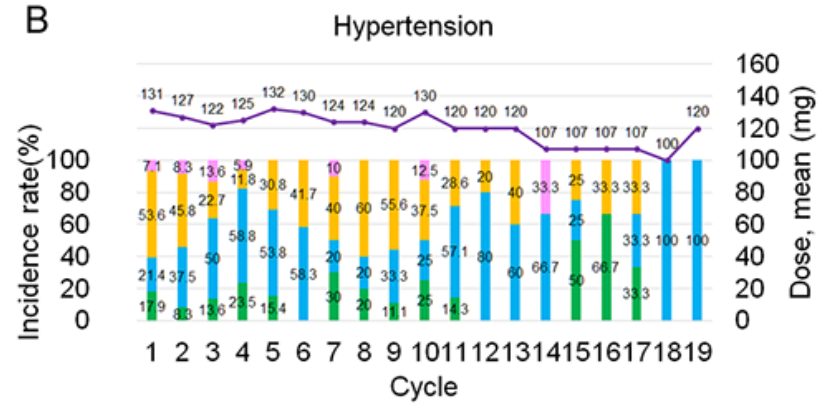

C
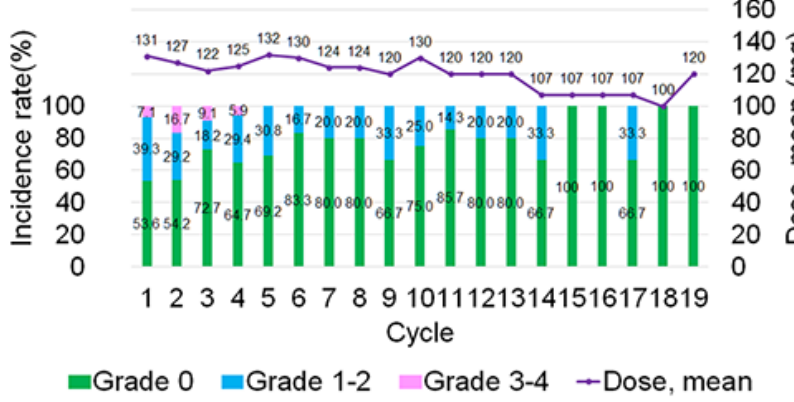

Figure 3. Toxicities of any grade (potentially associated with regorafenib) and mean dose of regorafenib occurring in the 12-month treatment period. (A) Hand-and-foot skin reactions. (B) Hypertension. (C) Hepatic toxicity.

Safety. The mean dose of regorafenib per day at 19 weeks was reduced to $120 \mathrm{mg}$, and $4 / 28$ patients managed to re-escalate the dose $(14.29 \%)$. Safety was assessed in all 28 patients. Despite the majority of patients requiring $\geq 1$ dose reduction due to toxicity, some patients $(4 / 28 ; 14.29 \%)$ subsequently had their regorafenib dose re-escalated without recurrence of unfavorable adverse effects. Particularly, the mean dose at 18 and 19 cycles of regorafenib per day was 100 and $120 \mathrm{mg}$, respectively, since $14.29 \%$ of patients re-escalated their dose. Table VI summarizes the hematological and non-hematological adverse events in patients. The leading grade 1-2 adverse events were hypertension $(20 / 28 ; 71.43 \%)$, anemia $(19 / 28 ; 67.86 \%)$ and hand-and-foot skin reactions (HFSRs; 18/28; 64.29\%; Fig. 3A). The leading grade 3 adverse events were HFSRs $(6 / 28 ; 21.43 \%)$, hypertension $(6 / 28 ; 21.43 \%)$ and hepatic toxicity $(5 / 28 ; 17.86 \%$; Fig. $3 \mathrm{~A})$.

\section{Discussion}

The present single-center study investigated treatment outcomes for patients with pre-treated metastatic GIST treated with regorafenib. Several points of interest were observed. Firstly, the median PFS and OS time for all 28 patients were 4.4 and 29.3 months, respectively. Regorafenib exhibited similar clinical efficacy for Taiwanese patients compared with the GRID trial comprising of Asian patients, such as Korean and Japanese, with advanced GIST who experienced treatment failure with imatinib or sunitinib (23-25). Secondly, regarding regorafenib-induced adverse events, all patients exhibited similar treatment-associated toxicity profiles compared with those of the previous phase II (26) and III GRID trials (16), but with a lower incidence of grade III hypertension and diarrhea (23 and 5\% in the phase III GRID trial vs. 21 and $0 \%$ in the present study, respectively). In addition, these adverse events corroborate with the toxicity profile of other kinase inhibitors with a similar target spectrum $(27,28)$. Since the dose had to be reduced and was then re-escalated in some patients, it was not possible to draw any conclusions regarding the possible dose-response associations between regorafenib and adverse events in the present study.

HFSR was the most frequently observed adverse event and the most common reason for dose reduction in the present study. Although HFSRs are not lethal adverse effects, these conditions are associated with substantial unfavorable clinical symptoms, such as intractable pain and dose reduction and treatment may be stopping (18). Previous studies have demonstrated that Asian patients are particularly susceptible to regorafenib-induced HFSRs $(18,25,29)$. The incidence of HFSR in the present study population (85.71\%) was higher compared with that in the regorafenib group in the GRID trial (56\%) (23) but was similar to Japanese subgroup (92\%) in the GRID trial (29). Genetic polymorphisms of TNF- $\alpha$, VEGF 


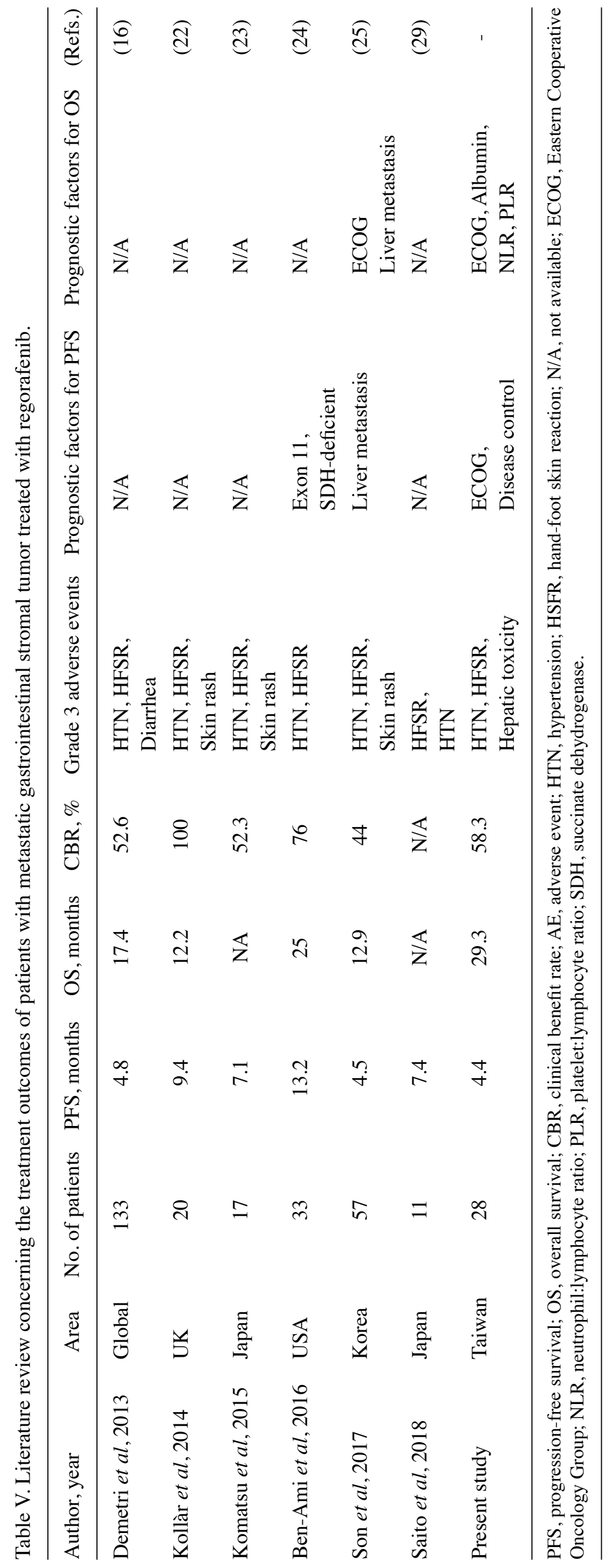


Table VI. Adverse events and laboratory abnormalities of 28 patients with gastrointestinal stromal tumor following regorafenib treatment at a starting dose of $160 \mathrm{mg}$.

\begin{tabular}{lccc}
\hline & & Grade, $\mathrm{n}(\%)$ & Grade 3 \\
\cline { 2 - 4 } Adverse effect & Any grade & Grade 1-2 & $15(53.57)$ \\
\hline Any event & $28(100.00)$ & $13(46.43)$ & $6(21.43)$ \\
Hypertension & $26(92.86)$ & $20(71.43)$ & $6(21.43)$ \\
Hand-and-foot skin reaction & $24(85.71)$ & $18(64.29)$ & $3(10.71)$ \\
Anemia & $22(78.57)$ & $19(67.86)$ & $5(17.86)$ \\
Hepatic toxicity & $15(53.57)$ & $10(35.71)$ & $1(3.57)$ \\
Thrombocytopenia & $9(32.14)$ & $8(28.57)$ & 0 \\
Fatigue & $8(28.57)$ & $8(28.57)$ & 0 \\
Diarrhea & $7(25.00)$ & $7(25.00)$ & 0 \\
Hypothyroidism & $6(21.43)$ & $6(21.43)$ & 0 \\
Hoarseness & $4(14.29)$ & $4(14.29)$ & 0 \\
Anorexia & $3(10.71)$ & $3(10.71)$ & 0 \\
Myalgia & $3(10.71)$ & $2(7.14)$ & 0 \\
Oral mucositis & $2(7.14)$ & $2(7.14)$ & 0 \\
Palpitation & $2(7.14)$ & $1(3.57)$ & 0 \\
Alopecia & $1(3.57)$ & 0 & 0 \\
Leukopenia & 0 & & \\
\hline
\end{tabular}

and UGT1A9 genes have been reported to be associated with the increased susceptibility of Asian patients to tyrosine kinase inhibitor-induced HFSRs, particularly in patients with hepatocellular carcinoma treated with sorafenib (18). Furthermore, the incidence of HFSRs in the present study was similar compared with that of Korean patients (82\%) (25). Studies investigating the underlying molecular mechanisms of this increased susceptibility to regorafenib-induced HSFRs are required.

Good performance status and disease control mediated by regorafenib were independent factors for a favorable PFS time in the present study, supporting a previous study demonstrating that good performance status was consistently and independently associated with favorable PFS and OS time (12). Korean and Japanese patients with GISTs, who displayed good performance status, also had improved PFS and OS time with regorafenib treatment $(23,25,29)$.

Regarding OS time, several novel prognostic factors were found in the present study, including liver metastasis, the pretreated albumin level, NLR and PLR. Similar to the Korean study (25), liver metastasis was a favorable factor for OS time, demonstrated by univariate survival analysis; however, liver metastasis was not an independent prognostic factor for OS time. Regarding the pretreated albumin level, a previous study reported higher pretreated serum albumin expression levels following two failed lines of TKIs in patients with pretreated metastatic GIST, and that these increased serum albumin expression levels were favorable factors associated with an improved OS time (30); however, in contrast with the present study, this previous study used nilotinib, sorafenib and imatinib as third-line TKIs, and therefore the results cannot be compared with those from the present study. In a meta-analysis including 29 studies investigating cancer of the gastrointestinal tract, 26/29 studies found that higher serum albumin levels were associated with improved survival using multivariate analysis (31). Therefore, further studies are required to resolve the molecular mechanisms underlying the aforementioned association so that increasing albumin levels may be used as a part of cancer treatment to improve OS time.

Previously, several studies demonstrated the association between the inflammatory and immunonutritional status and the prognosis of patients with cancer, including NLR and PLR (32-36). A high NLR was associated with poor prognosis in several malignancies, including pancreatic cancer, hepatocellular carcinoma, ovarian cancer and GIST (33-36). Although elevated NLR and PLR have been reported to be associated with poor treatment outcomes, including PFS and OS time, for primary GIST (37-41), to the best of our knowledge, the present study is the first study to demonstrate the association between lower NLR and PLRs to a more favorable OS time in patients with pretreated metastatic GIST receiving regorafenib.

The mechanism underlying elevated NLR and poor prognosis in GISTs is still unknown; however, elevated NLR usually indicates an imbalance between pro-tumor and the anti-tumor immune responses (42-44). Lymphocytes inhibit the proliferation and metastatic ability of cancer cells by inducing cytotoxic effects and cytokines production $(45,46)$. Neutrophils have been demonstrated to induce tumor proliferation, invasion and vascularization by releasing proangiogenic chemokines (47-49), therefore increased neutrophils can inhibit the immune system by suppressing the cytolytic activity of immune cells, such as lymphocytes and nature killer cells $(50,51)$. Thus, an elevated NLR directs the aforementioned imbalance in favor of the pro-tumor inflammatory status, which in turn causes an unfavorable outcome. 
PLR has been reported as a poor prognostic factor in ovarian (52), colorectal (53), esophageal (54), pancreatic (55), endometrial cancer (56) and neuroendocrine tumors (57), as well as in primary GIST (40). A high PLR range between 150 and 300 is associated with less favorable outcomes, in terms of recurrence-free survival, cancer-specific survival or OS time (40). Inflammation has been recognized to be positively associated with PFS and OS outcomes of malignancy and is a contributor to the shutdown of the anti-tumor immune response by activating mediating $\mathrm{T}$ cells and chemokines release, facilitating tumor growth and metastasis (58). A non-specific response to cancer-associated inflammation was represented by the presence of neutrophilia and thrombocytosis (40). However, both the underlying mechanism, which links leukocytosis and neutrophilia to the progression of malignant tumors and explains the increase in platelets, and the biological pro-inflammatory behavior of cancer cells, remain unclear (47).

Overall, for Taiwanese patients with pre-treated GIST treated with regorafenib, poor performance status and poor disease control predicted an unfavorable PFS time; however, poor performance status, high NLR, PLR and low serum albumin levels predicted an unfavorable OS time.

\section{Acknowledgements}

Not applicable.

\section{Funding}

No funding was received.

\section{Availability of data and materials}

The datasets used and/or analyzed during the present study are available from the corresponding author on reasonable request.

\section{Authors' contributions}

$\mathrm{CHH}$ collected the data and reviewed the literature. CNY designed the study, collected the data and wrote the manuscript. JSC, CYT, SYW, CTC and TSY interpreted the data and critically revised the manuscript for important intellectual content. $\mathrm{CHH}$ and $\mathrm{CNY}$ analyzed the data. All authors read and approved the final manuscript.

\section{Ethics approval and consent to participate}

This study protocol was approved by The Institutional Review Board of the Chang Gung Memorial Hospital (Taoyuan, Taiwan; approval no. 103-6044A3). Written informed consent was provided by all patients.

\section{Patient consent for publication}

Not applicable.

\section{Competing interests}

The authors declare that they have no competing interests.

\section{References}

1. Tzen CY, Wang JH, Huang YJ, Wang MN, Lin PC, Lai GL, Wu CY and Tzen CY: Incidence of gastrointestinal stromal tumor: A retrospective study based on immunohistochemical and mutational analyses. Dig Dis Sci 52: 792-797, 2007.

2. Blanke CD and Corless CL: State-of-the art therapy for gastrointestinal stromal tumors. Cancer Invest 23: 274-280, 2005.

3. Hirota S, Isozaki K, Moriyama Y, Hashimoto K, Nishida T, Ishiguro S, Kawano K, Hanada M, Kurata A, Takeda M, et al: Gain-of-function mutations of c-kit in human gastrointestinal stromal tumors. Science 279: 577-580, 1998.

4. Druker BJ, Tamura S, Buchdunger E, Ohno S, Segal GM, Fanning S, Zimmermann J and Lydon NB: Effects of a selective inhibitor of the Abl tyrosine kinase on the growth of Bcr-Abl positive cells. Nat Med 2: 561-566, 1996.

5. Buchdunger E, Cioffi C, Law N, Stover D, Ohno-Jones S, Druker BJ and Lydon NB: Abl protein-tyrosine kinase inhibitor STI571 inhibits in vitro signal transduction mediated by c-kit and platelet-derived growth factor receptors. J Pharmacol Exp Ther 295: 139-145, 2000.

6. Heinrich MC, Griffith DJ, Druker BJ, Wait CL, Ott KA and Zigler AJ: Inhibition of c-kit receptor tyrosine kinase activity by STI 571, a selective tyrosine kinase inhibitor. Blood 96: 925-932, 2000.

7. Wang WL, Healy ME, Satter M, Verma S, Lin J, Maulik G, Stiles CD, Griffin JD, Johnson BE and Salgia R: Growth inhibition and modulation of kinase pathways of small cell lung cancer cell lines by the novel tyrosine kinase inhibitor STI 571. Oncogene 19: 3521-3528, 2000.

8. Joensuu H, Roberts PJ, Sarlomo-Rikala M, Andersson LC, Tervahartiala P, Tuveson D, Silberman S, Capdeville R, Dimitrijevic S, Druker B and Demetri GD: Effect of the tyrosine kinase inhibitor STI571 in a patient with a metastatic gastrointestinal stromal tumor. N Engl J Med 344: 1052-1056, 2001.

9. Tuveson DA, Willis NA, Jacks T, Griffin JD, Singer S, Fletcher CD, Fletcher JA and Demetri GD: STI571 inactivation of the gastrointestinal stromal tumor c-KIT oncoprotein: Biological and clinical implications. Oncogene 20: 5054-5058, 2001.

10. Demetri GD, von Mehren M, Blanke CD, Van den Abbeele AD, Eisenberg B, Roberts PJ, Heinrich MC, Tuveson DA, Singer S, Janicek M, et al: Efficacy and safety of imatinib mesylate in advanced gastrointestinal stromal tumors. N Engl J Med 347: 472-480, 2002.

11. Blanke CD, Demetri GD, von Mehren M, Heinrich MC, Eisenberg B, Fletcher JA, Corless CL, Fletcher CD, Roberts PJ, Heinz D, et al: Long-term results from a randomized phase ii trial of standard-versus higher-dose imatinib mesylate for patients with unresectable or metastatic gastrointestinal stromal tumors expressing kit. J Clin Oncol 26: 620-625, 2008

12. Yeh CN, Chen TW, Lee HL, Liu YY, Chao TC, Hwang TL, Jan YY and Chen MF: Kinase mutations and imatinib mesylate response for 64 Taiwanese with metastatic GIST: Preliminary experience from Chang Gung Memorial Hospital. Ann Surg Oncol 14: 1123-1128, 2007.

13. Demetri GD, von Oosterom AT, Garrett CR, Blackstein ME, Shah MH, Verweij J, McArthur G, Judson IR, Heinrich MC, Morgan JA, et al: Efficacy and safety of sunitinib in patients with advanced gastrointestinal stromal tumour after failure of imatinib: A randomised controlled trial. Lancet 368: 1329-1338, 2006.

14. Chen YY, Yeh CN, Cheng CT, Chen TW, Rau KM, Jan YY and Chen MF: Sunitinib for Taiwanese patients with gastrointestinal stromal tumor after imatinib treatment failure or intolerance. World J Gastroenterol 17: 2113-2119, 2011.

15. Wilhelm SM, Dumas J, Adnane L, Lynch M, Carter CA, Schütz G, Thierauch KH and Zopf D: Regorafenib (BAY 73-4506): A new oral multikinase inhibitor of angiogenic, stromal and oncogenic receptor tyrosine kinases with potent preclinical antitumor activity. Int J Cancer 129: 245-255, 2011.

16. Demetri GD, Reichardt P, Kang YK, Blay JY, Rutkowski P, Gelderblom H, Hohenberger P, Leahy M, von Mehren M, Joensuu $\mathrm{H}$, et al: Efficacy and safety of regorafenib for advanced gastrointestinal stromal tumours after failure of imatinib and sunitinib (GRID): An international, multicentre, randomised, placebo-controlled, phase 3 trial. Lancet 26: 295-302, 2013.

17. ESMO/European Sarcoma Network Working Group. Gastrointestinal stromal tumours: ESMO Clinical Practice Guidelines for diagnosis, treatment and follow-up. Ann Oncol 25 (Suppl 3): iii21-iii26, 2014. 
18. Yeh CN, Chen MH, Chen YY, Yang CY, Yen CC, Tzen CY, Chen LT and Chen JS: A phase II trial of regorafenib in patients with metastatic and/or an unresectable gastrointestinal stromal tumor harboring secondary mutations of exon 17. Oncotarget 8: 44121-44130, 2017.

19. Eisenhauer EA, Therasse P, Bogaerts J, Schwartz LH, Sargent D, Ford R, Dancey J, Arbuck S, Gwyther S, Mooney M, et al: New response evaluation criteria in solid tumours: Revised RECIST guideline (version 1.1). Eur J Cancer 42: 228-247, 2009.

20. https://evs.nci.nih.gov/ftp1/CTCAE/CTCAE_4.03/CTCAE_4.03 2010-06-14_QuickRefe nce_8.5x11.pdf (Accessed on June 14, 2010).

21. Heinrich MC, Corless CL, Demetri GD, Blanke CD, von Mehren M, Joensuu H, McGreevey LS, Chen CJ, Van den Abbeele AD, Druker BJ, et al: Kinase mutations and imatinib response in patients with metastatic gastrointestinal stromal tumor. J Clin Oncol 21: 4342-4349, 2003.

22. Kollàr A, Maruzzo M, Messiou C, Cartwright E, Miah A, Martin-Liberal J, Thway K, McGrath E, Dunlop A, Khabra K, et al: Regorafenib treatment for advanced, refractory gastrointestinal stromal tumor: A report of the UK managed access program. Clin Sarcoma Res 4: 17, 2014.

23. Komatsu Y, Doi T, Sawaki A, Kanda T, Yamada Y, Kuss I, Demetri GD and Nishida T: Regorafenib for advanced gastrointestinal stromal tumors following imatinib and sunitinib treatment: A subgroup analysis evaluating Japanese patients in the phase III GRID trial. Int J Clin Oncol 20: 905-912, 2015.

24. Ben-Ami E, Barysauskas CM, von Mehren M, Heinrich M, Corless CL, Butrynski JE, Morgan JA, Wagner AJ, Choy E, Yap JT, et al: Long-term follow-up results of the multicenter phase II trial of regorafenib in patients with metastatic and/or unresectable GI stromal tumor after failure of standard tyrosine kinase inhibitor therapy. Ann Oncol 27: 1794-1799, 2016.

25. Son MK, Ryu MH, Park JO, Im SA, Kim TY, Lee SJ, Ryoo BY, Park SR and Kang YK: Efficacy and safety of regorafenib in korean patients with advanced gastrointestinal stromal tumor after failure of imatinib and sunitinib: A multicenter study based on the management access program. Cancer Res Treat 49: 350-357, 2017.

26. George S, Wang Q, Heinrich MC, Corless CL, Zhu M, Butrynski JE Morgan JA, Wagner AJ, Choy E, Tap WD, et al: Efficacy and safety of regorafenib in patients with metastatic and/or unresectable GI stromal tumor after failure of imatinib and sunitinib: A multicenter phase II trial. J Clin Oncol 30: 2401-2407, 2012.

27. Llovet JM, Ricci S, Mazzaferro V, Hilgard P, Gane E, Blanc JF, de Oliveira AC, Santoro A, Raoul JL, Forner A, et al: (SHARP Investigators Study Group). Sorafenib in advanced hepatocellular carcinoma. N Engl J Med 359: 378-390, 2008.

28. Cheng AL, Kang YK, Chen Z, Tsao CJ, Qin S, Kim JS, Luo R, Feng J, Ye S, Yang TS, et al: Efficacy and safety of sorafenib in patients in the Asia-pacific region with advanced hepatocellular carcinoma: A phase III randomised, double-blind, placebo-controlled trial. Lancet Oncol 10: 25-34, 2009.

29. Saito Y, Takahashi T, Tanaka K, Miyazaki Y, Makino T, Kurokawa Y, Yamasaki M, Nakajima K, Takiguchi S, Mori M and Doki Y: Treatment of regorafenib in patients with metastatic or unresectable gastrointestinal stromal tumor after failure of imatinib and sunitinib. Gan To Kagaku Ryoho 45: 121-123, 2018 (In Japanese).

30. Italiano A, Cioffi A, Coco P, Maki RG, Schöffski P, Rutkowski P, Le Cesne A, Duffaud F, Adenis A, Isambert N, et al: Patterns of care, prognosis, and survival in patients with metastatic gastrointestinal stromal tumors (GIST) refractory to first-line imatinib and second-line sunitinib. Ann Surg Oncol 19: 1551-1559, 2012.

31. Gupta D and Lis CG: Pretreatment serum albumin as a predictor of cancer survival: A systematic review of the epidemiological literature. Nutr J 9: 69, 2010.

32. Proctor MJ, Morrison DS, Talwar D, Balmer SM, O'Reilly DS, Fletcher CD, Foulis AK, Horgan PG and McMillan DC: A comparison of inflammation-based prognostic scores in patients with cancer. A Glasgow Inflammation Outcome Study. Eur J Cancer 47: 2633-2641, 2011.

33. Glazer ES, Rashid OM, Pimiento JM, Hodul PJ and Malafa MP: Increased neutrophil-to-lymphocyte ratio after neoadjuvant therapy is associated with worse survival after resection of borderline resectable pancreatic ductal adenocarcinoma. Surgery 160: 1288-1293, 2016

34. Goh BK, Kam JH, Lee SY, Chan CY, Allen JC, Jeyaraj P, Cheow PC, Chow PK, Ooi LL and Chung AY: Significance of neutrophil-to-lymphocyte ratio, platelet-to-lymphocyte ratio and prognostic nutrition index as preoperative predictors of early mortality after liver resection for huge $(>/=10 \mathrm{~cm})$ hepatocellular carcinoma. J Surg Oncol 113: 621-627, 2016.
35. Dobrzycka B, Mackowiak-Matejczyk B, Terlikowska KM, Kulesza-Bronczyk B, Kinalski M and Terlikowski SJ: Serum levels of IL-6, IL-8 and CRP as prognostic factors in epithelial ovarian cancer. Eur Cytokine Netw 24: 106-113, 2013.

36. Perez DR, Baser RE, Cavnar MJ, Balachandran VP, Antonescu CR, Tap WD, Strong VE, Brennan MF, Coit DG, Singer S and Dematteo RP: Blood neutrophil-to-lymphocyte ratio is prognostic in gastrointestinal stromal tumor. Ann Surg Oncol 20: 593-599, 2013.

37. Jiang C, Hu WM, Liao FX, Yang Q, Chen P, Rong YM, Guo GF, Yin CX, Zhang B, He WZ and Xia LP: Elevated preoperative neutrophil-to-lymphocyte ratio is associated with poor prognosis in gastrointestinal stromal tumor patients. Onco Targets Ther 9: $877-883,2016$

38. Xue A, Gao X, Fang Y, Shu P, Ling J, Shen K, Sun Y, Qin J, Qin X and Hou Y: Incorporation of NLR into NIH stratification system increases predictive accuracy for surgically resected gastrointestinal stromal tumors. Acta Biochim Biophys Sin (Shanghai) 49: 179-185, 2016

39. Kumamoto Y, Kaizu T, Tajima H, Nishizawa N, Ei S, Igarashi K and Watanabe M: Neutrophil-to-lymphocyte ratio as a predictor of postoperative morbidity in patients with distal cholangiocarcinoma. Mol Clin Oncol 9: 362-368, 2018.

40. Racz JM, Cleghorn MC, Jimenez MC, Atenafu EG, Jackson TD, Okrainec A, Venkat Raghavan L and Quereshy FA: Predictive ability of blood neutrophil-to-lymphocyte and platelet-to-lymphocyte ratios in gastrointestinal stromal tumors. Ann Surg Oncol 22: 2343-2350, 2015.

41. Goh BK, Chok AY, Allen JC Jr, Quek R, Teo MC, Chow PK, Chung AY, Ong HS and Wong WK: Blood neutrophil-to-lymphocyte and platelet-to-lymphocyte ratios are independent prognostic factors for surgically resected gastrointestinal stromal tumors. Surgery 159: 1146-1156, 2016.

42. An X, Ding PR, Li YH, Wang FH, Shi YX, Wang ZQ, He YJ, $\mathrm{Xu}$ RH and Jiang WQ: Elevated neutrophil to lymphocyte ratio predicts survival in advanced pancreatic cancer. Biomarkers 15: 516-522, 2010.

43. Shibutani M, Maeda K, Nagahara H, Noda E, Ohtani H, Nishiguchi $Y$ and Hirakawa $\mathrm{K}$ : A high preoperative neutrophil-to-lymphocyte ratio is associated with poor survival in patients with colorectal cancer. Anticancer Res 33: 3291-3294, 2013.

44. Li X, Chen ZH, Ma XK, Chen J, Wu DH, Lin Q, Dong M, Wei L, Wang TT, Ruan DY, et al: Neutrophil-to-lymphocyte ratio acts as a prognostic factor for patients with advanced hepatocellular carcinoma. Tumor Biol 35: 11057-11063, 2014.

45. Ownby HE, Roi LD, Isenberg RR and Brennan MJ: Peripheral lymphocyte and eosinophil counts as indicators of prognosis in primary breast cancer. Cancer 52: 126-130, 1983.

46. Terzić J, Grivennikov S, Karin E and Karin M: Inflammation and colon cancer. Gastroenterology 138: 2101-2114.e5, 2010.

47. Shamamian P, Schwartz JD, Pocock BJ, Monea S, Whiting D, Marcus SG and Mignatti P: Activation of progelatinase A (MMP-2) by neutrophil elastase, cathepsin G, and proteinase-3: A role for inflammatory cells in tumor invasion and angiogenesis. J Cell Physiol 189: 197-206, 2001.

48. Gong Y and Koh DR: Neutrophils promote inflammatory angiogenesis via release of preformed VEGF in an in vivo corneal model. Cell Tissue Res 339: 437-448, 2010.

49. Neagoe PE, Brkovic A, Hajjar F and Sirois MG: Expression and release of angiopoietin-1 from human neutrophils: Intracellular mechanisms. Growth Factors 27: 335-344, 2009.

50. Müller I, Munder M,Kropf P and Hansch GM: Polymorphonuclear neutrophils and T lymphocytes: Strange bedfellows or brothers in arms? Trends Immunol 30: 522-530, 2009.

51. el-Hag A and Clark RA: Immunosuppression by activated human neutrophils. Dependence on the myeloperoxidase system. J Immunol 139: 2406-2413, 1987.

52. Asher V, Lee J, Innamaa A and Bali A: Preoperative platelet-lymphocyte ratio as an independent prognostic marker in ovarian cancer. Clin Transl Oncol 13: 499-503, 2011.

53. Kwon HC, Kim SH, Oh SY, Lee S, Lee JH, Choi HJ, Park KJ, Roh MS, Kim SG, Kim HJ and Lee JH: Clinical significance of preoperative neutrophil-lymphocyte versus platelet-lymphocyte ratio in patients with operable colorectal cancer. Biomarkers 17: 216-222, 2012

54. Feng JF, Huang Y, Zhao Q and Chen QX: Clinical significance of preoperative neutrophil-lymphocyte ratio versus platelet-lymphocyte ratio in patients with small cell carcinoma of the esophagus. ScientificWorldJournal 2013: 504365, 2013. 
55. Smith RA, Bosonnet L, Raraty M, Sutton R, Neoptolemos JP, Campbell F and Ghaneh P: Preoperative platelet-lymphocyte ratio is an independent significant prognostic marker in resected pancreatic ductal adenocarcinoma. Am J Surg 197: 466-472, 2009.

56. Wang D, Yang JX, Cao DY, Wan XR, Feng FZ, Huang HF Shen K and Xiang Y: Preoperative neutrophil-lymphocyte and platelet-lymphocyte ratios as independent predictors of cervical stromal involvement in surgically treated endometrioid adenocarcinoma. Onco Targets Ther 6: 211-216, 2013.

57. Sakka N, Smith RA, Whelan P, Ghaneh P, Sutton R, Raraty M, Campbell F and Neoptolemos JP: A preoperative prognostic score for resected pancreatic and periampullary neuroendocrine tumours. Pancreatology 9: 670-676, 2009.
58. Bhatti I, Peacock O, Lloyd G, Larvin $M$ and Hall RI: Preoperative hematologic markers as independent predictors of prognosis in resected pancreatic ductal adenocarcinoma: Neutrophil-lymphocyte versus platelet-lymphocyte ratio. Am J Surg 200: 197-203, 2010.

(c) (i) (5) This work is licensed under a Creative Commons Attribution-NonCommercial-NoDerivatives 4.0 International (CC BY-NC-ND 4.0) License. 